\title{
Automatic Characterization of Ambulatory Patterns of Utilitarian and Leisure Trips
}

\author{
Aki Härmä and Koen de Groot \\ Philips Research \\ Eindhoven, The Netherlands
}

\begin{abstract}
In the health self-management services it is beneficial to identify and address the already existing healthy activity patterns of the user. Some of these healthy activity patterns might be of a utilitarian nature, e.g. commuting to work by bike or on foot, or might be for leisure, like taking a walk in a park. In this paper we discuss one possibility to detect the utilitarian or leisure nature of a particular ambulatory path based on the geometry of the trajectory. In essence, a leisure trip is more commonly a round-trip while an utilitarian A-to-B trips follow the single shortest path between $A$ and $B$. We define a generic measure for the characterization of utilitarian and leisure paths based on GPS location data and develop an algorithm for approaching the same based on only magnetometer data from a wearable device.
\end{abstract}

\section{INTRODUCTION}

When we go to work or visit a supermarket most of us are likely to choose the shortest route to the destination and back. On the other hand, a person having a walk for health purposes often prefers choosing a path around an area, for example, a nearby park or a block. This paper is based on this common observation that some geometric property of a path may be associated with the utilitarian or leisure character of a trip the person takes. Experiments by [1] show clear geographic and cultural differences but there is objective evidence that utilitarian commuters typically choose the shortest route from A to $\mathrm{B}[2]$.

It is commonly understood that lifestyle is one of the most important determinants of overall health, see, e.g., [3]. Walking is a healthy and safe form of physical activity and therefore it is often recommended in health programs aiming at increasing the physical activity level of the user [4]. Walking is also easy to measure using, e.g., pedometers, bracelets, and apps [5], [6]. However, changing a lifestyle by adding new active routines like healthy walks is not easy because of various economical, social, and environmental constraints. Therefore, health coaches often try to identify healthy routines the customer already has and then ask the user to perform them more often or make them more intense. In automated health self-management programs where communication is based on sensor data, it is not straightforward to know which routines are healthy routines that can be boosted. People are not ready to commute more often or extend a trip to a supermarket. On the contrary, a healthy walk around a park can be repeated more often or extended when the subject has the motivation for it and understands the health benefits of it.
In this paper it is assumed that a geometric property of the path may give an indirect indication of the purpose of the trip. In particular, we assume that a trip may be an utilitarian trip from a place A to B and back the shortest path, or a healthy or leisure activity bout with a path that encloses a geographic area. The path circularity measure introduced in the following section is based on accurate geographic position data. In absence of position data, the detection of the geometry is of course more challenging. However, there are possibilities to use various sensor modalities to detect if the user took the same or different route when returning. There is anecdotal evidence on how pets find a way back to home over long distances, how migratory birds [7], [8] return back to the nesting sites, a salmon finds the way back to the same creek where it hatched [9], or rats learn paths in mazes [10].

Bio-inspired positioning based on local magnetic signatures has been proposed in [11]. In the current paper this is extended to the problem of detection of the return path. The ability of animals to navigate a way back without a dedicated external positioning infrastructure is a good model for the design of data processing also in low-power wearable devices. In section III, which is inspired by this observation, we focus on deriving geometric properties from trips solely based on data output by low-power magnetic sensors. For this we introduce two algorithms for the identification of return path from a two-way trip. Finally, the properties of the proposed measure and the two algorithms are demonstrated using test data from wearable sensors collected during cycling and walking trips.

\section{Circularity IN POSITION DATA}

Many personal health products and services come with an app which tracks the location of the user by means of global positioning techniques that rely on satellites or other beacons. A location path can be represented by discrete time-series of geographic points $\mathbf{g}(t)=\left[g_{x}(t), g_{y}(t), g_{z}(t)\right]^{T}$ corresponding to the latitude, longitude, and elevation, respectively. The length of the path can be estimated by

$$
L=\sum_{t=0}^{T-1}|\mathbf{g}(t)-\mathbf{g}(t-1)|
$$

There are several practical approaches also to compute the area enclosed by the path. On a plane, one may use the popular shoelace algorithm, see, e.g., [12]. The method works well 
with clean GPS data and in the current notation it can be written by

$$
\begin{aligned}
A= & \frac{1}{2} \mid \sum_{t=0}^{T-1} g_{x}(t) g_{y}(t+1)+g_{x}(T) g_{y}(0) \\
& -\sum_{t=0}^{T-1} g_{x}(t+1) g_{y}(t)-g_{x}(0) g_{y}(T) \mid
\end{aligned}
$$

Segmented areas in binary images are sometimes characterized by the ratio of the circumference to the area of a blob. see, e.g. [13]. In this paper we use a very similar normalized circularity measure for the path data. The measure is given by

$$
C=4 \pi \frac{A}{L^{2}}
$$

When the path is a full circle the formula gives the maximum value $C=1.0$, while for any other shape, the value is smaller. The minimum $C=0$ is obtained when the enclosed area $A=0$, that is, the same path was used in both ways on a visit from $\mathrm{A}$ to $\mathrm{B}$ and back.

Figures 1 show histograms of walking, running, and cycling trips by a group of 85 volunteers who were wearing an activity tracking watch and an app with GPS localization in an experiment lasting several weeks. Only semi-continuous trips longer than one kilometer, with the start and end locations in close proximity, were included in the data set. Both walking and cycling trips contain significant number of utilitarian ABA trips where $C \approx 0$. In running data most of the trips had a clear circular pattern, although, the running trips were not very popular in this population of 85 subjects. As reference, the histogram of transportation trips is shown. Most of the trajectories classified as transportation event have a clear utilitarian ABA trip pattern. Based on examination of the location data it became clear that a large number of cycling trips were indeed utilitarian visits to a supermarket or a work place.
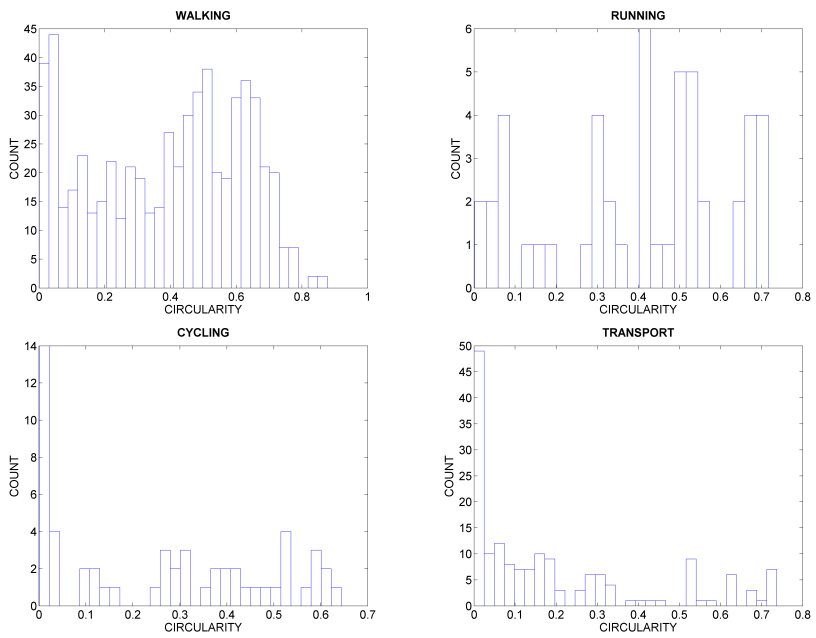

Fig. 1: Circularity $C$ in a collection of walking, running, cycling and transport trips in 85 individuals.

\section{CIRCULARITY IN MOTION SENSOR DATA}

Location data is typically not available in low-power wearable devices or indoors. However, some information about the path can be also estimated from elementary inertial sensors. In principle, it is possible to reconstruct a movement path by double-integrating the accelerometer data. This often has a significant drift due to sensor noise, nonlinearities, and other artifacts. Magnetometer sensors use the magnetic field of the Earth and therefore have a stable allocentric reference direction. Localization systems combining inertial and magnetic sensors have been proposed for example in [14], [15].

If the path on the way back from $B$ to $A$ is similar to the $\mathrm{AB}$ path, the enclosed area will be zero, and consequently the circularity $C \rightarrow 0$. One may say that the estimation of the lower bound of circularity is coupled to the problem of finding the return route, the task where many animals are very good at. Finding the return route requires memory and the ability to compare the current place to a memory signature. A typical approach would be to compute some global correlation metric between the two paths. It is often considered that humans and other animals are able to remember the order of historical events [16], and construct some cognitive spatial maps of locations [10] which support this processing principle. However, there is evidence that cognitive spatial information in human (and non-humans) is organized as relations between local contextual sub-maps rather than using a global geographic framework [17]. This suggests an alternative processing model where the correlations are computed between local segments.

\section{Global return path tracking}

The sensor data is a vector-values time series $\mathbf{x}(\mathbf{t})$. We assume that when the sensor moves from $\mathrm{A}$ to $\mathrm{B}$ and returns to A using the same path, the data corresponding to the path $\mathrm{BA}$ is a rotated and temporally reversed and distorted version of the time-series collected in AB. The sensor data for the entire trip can be modeled as follows:

$$
\mathbf{y}(\mathbf{t})=\left\{\begin{array}{l}
\mathbf{x}(\mathbf{t}), \text { if }, \mathbf{t}<\mathbf{T}_{\mathbf{B}} \\
M \mathbf{x}(\nu(\mathbf{t})), \text { if }, \mathbf{t} \geq \mathbf{T}_{\mathbf{B}}
\end{array}\right.
$$

where $M$ is a rotation matrix and $\nu()$ is a time warping function which is typically monotonically decreasing, i.e., mapping backwards in time. In a simplified case one may assume that the return path $x_{b}$ is simply a time reversal of the forward path, denoted $x_{a}$. In matrix notation the time-reversed path is then $M \overleftarrow{I} \mathbf{x}_{\mathbf{b}}$, where $\overleftarrow{I}$ is a reversal identity matrix and $\mathbf{x}_{\mathbf{b}}$ is the return part of the path. The goal is to minimize the loss

$$
e=\left(M \overleftarrow{I} \mathbf{x}_{\mathbf{b}}-\mathbf{x}_{\mathbf{a}}\right)^{2}
$$

The least-squares solution for the rotation matrix $M$ can be found from the normal equations and is written by

$$
\left(\overleftarrow{I} \mathbf{x}_{\mathbf{b}}\right)^{\mathbf{T}} \overleftarrow{I} \mathbf{x}_{\mathbf{b}} \mathbf{M}=\left(\overleftarrow{I} \mathbf{x}_{\mathbf{b}}\right)^{\mathbf{T}} \mathbf{x}_{\mathbf{a}}
$$

which reduces to

$$
\mathbf{x}_{\mathbf{b}}^{\mathbf{T}} \mathbf{x}_{\mathbf{b}} \mathbf{M}=\mathbf{x}_{\mathbf{b}}^{\mathbf{T}} \overleftarrow{I} \mathbf{x}_{\mathbf{a}}
$$


The ability to trace back the path requires the temporal reversal of the first path, but also a rotation $M$ of the sensory data because the orientation of the body in relation to the external field is different on the way back. A turn to the left in one way is replaced by a turn to the right on the way back.

In the current paper the temporal mid-point is used as an estimate of $T_{B}$, because the start and end points are known and a robust blind estimate of $T_{B}$ cannot be defined in a unique way in the case of loop data.

The detection of an estimate for the lower bound of circularity can be performed using the following algorithm:

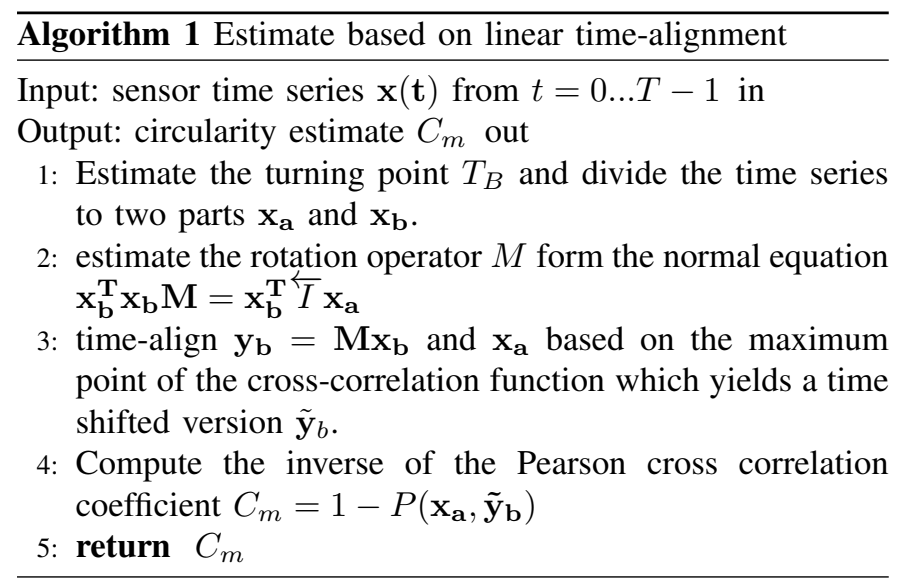

The outcome of the algorithm is a measure $C_{m}$ which gives a small value when the data in the two ways has the assumed time-warped temporal and geometric rotation, and a larger value, when the similarity is low. If the return path is a symmetrical mirror image of the $\mathrm{AB}$ path (e.g., in a perfect circle or square path), it is possible to find a rotation matrix $R$ which gives a large value for $C_{m}$. In real movement data, perfect mirror-symmetrical paths are unusual.

\section{Return path matching using local dynamic time-warping}

The pace on the way back may be different in different parts of the path which cannot be compensated for by the time-alignment operation in the global path matching algorithm above, where $\nu(t)$ was merely a time-reversal and shift function. A generic (reversed) time-warping function can be estimated using various methods for dynamic time warping (DTW) [18]. These methods are typically based on a piecewise linear time-warping function $\nu(t)$ matched to the data. One can note that this is conceptually similar to the biological mechanism of remembering the return path as sequence of local contextual sub-maps. The algorithm can be written as follows:

The following experiments were based on the DWT implementation available in the Matlab Signal Processing Toolbox $2016 \mathrm{~b}$ which is based on the method detailed in [18].

\section{EXPERIMENTS}

The test data contains multisensor (Shimmer3) measurements of a cyclist riding $1-3 \mathrm{~km}$ loops and two-way trips in a suburban area close to Eindhoven, The Netherlands. The
Algorithm 2 Estimate based on dynamic time-warping

Input: sensor time series $\mathbf{x}(\mathbf{t})$ from $t=0 \ldots T-1$ in

Output: circularity estimate $C_{d}$ out

1: Estimate the turning point $T_{B}$ and divide the time series to two parts $\mathbf{x}_{\mathbf{a}}$ and $\mathbf{x}_{\mathbf{b}}$.

2: estimate the rotation operator $M$ form the normal equation $\mathbf{x}_{\mathbf{b}}^{\mathbf{T}} \mathbf{x}_{\mathbf{b}} \mathbf{M}=\mathbf{x}_{\mathbf{b}}^{\mathbf{T}} \overleftarrow{I} \mathbf{x}_{\mathbf{a}}$

3: Find and optimal dynamic time-warping function $\nu(t)$ that minimize a norm of $\mathbf{y}_{\mathbf{b}}-\tilde{\mathbf{y}}_{\mathbf{b}}$, where $\tilde{\mathbf{y}}_{b}=\nu\left(M \mathbf{x}_{\mathbf{b}}\right)$. Typically DFT algorithms are based on a least squares norm.

4: Compute the inverse of the Pearson cross correlation coefficient $C_{d}=1-P\left(\mathbf{x}_{\mathbf{a}}, \tilde{\mathbf{y}}_{\mathbf{b}}\right)$

5: return $C_{d}$

sensor contains two 3-axis accelerometer devices, gyroscope, pressure sensor, and a 3 -axis magnetometer. The device was attached to the chest of the cyclist using an elastic strap. The GPS location data was collected using an app in a smartphone carried by the cyclist. In total, 14 loops or two-way trips were recorded. Examples of the cycling paths are shown in Fig. 2. The measures of circularity based on the location data $(C)$ and the magnetometer data $C_{m}$ are marked in the figures.
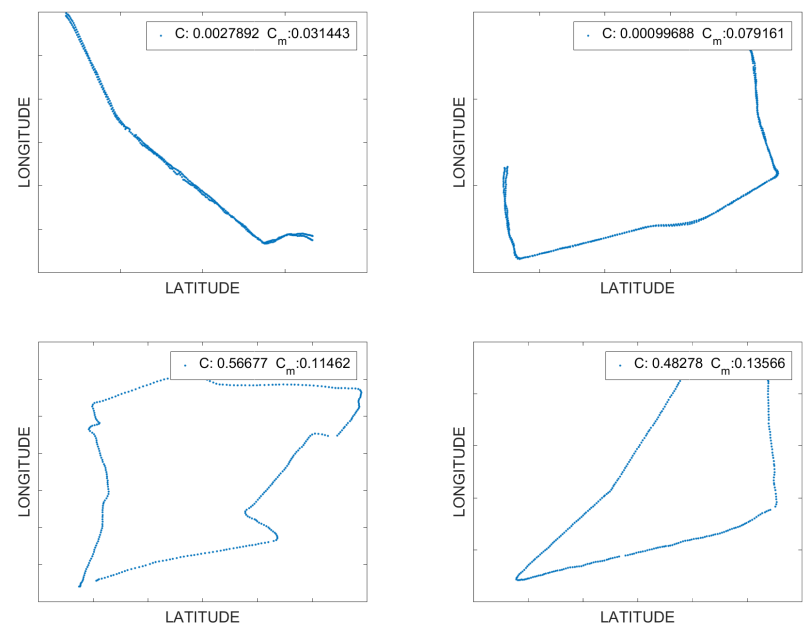

Fig. 2: Examples of cycling path trajectories.

An example of raw magnetometer data from an $\mathrm{AB}$ trip is shown in the top panel of Fig. 3. The return trip from B to A is shown in the middle panel and the rotated and time-aligned $\mathbf{y}_{\mathbf{b}}$ signal of the BA trip is in the bottom panel. The bottom and top panels are visually similar which is also reflected in the circularity estimate $C_{m}=0.05$.

The box plot of the circularity measure, derived from the collection of location and magnetometer data of loops and two-way trips in cycling and walking data, is shown in Fig. 4. In the cycling case there are three pairs of box plots represent the two kinds of trips using GPS data, global time alignment (TA) only, and the signals matched using DTW. The difference 

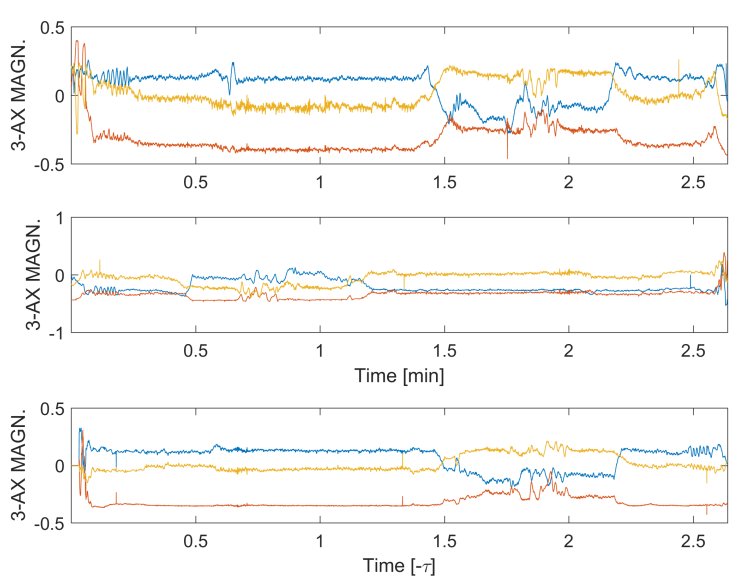

Fig. 3: Top) Magnetometer time-series of a cycling trip AB. Middle) from BA, and bottom) rotated and time-aligned BA data.

in the GPS and two other conditions is significant with a tiny $p$-value in all cases $\left(p<10^{-5}\right)$. Between the TA and DTW conditions there is a mild trend for the benefit of the DTW method but the difference is not significant. The bottom figure of Fig. 4 shows the circularity estimates for 8 ABA and 5 loop walking trips. The walking data was collected with the sensor attached to the belt of the subject which led to large movement artifacts in the magnetometer data. In this case the difference in the circularity estimate is only weakly significant in using linear temporal alignment $(p=0.07)$ but there is a benefit from using the DTW method where we get a significant difference with a $p$-value of $p=0.007$. The same experiment was also performed using accelerometer, gyroscope, temperature and air pressure data. However, the results in the experiments were less convincing and the difference in $C_{m}$ was significantly smaller than in the magnetometer data. However, the estimate for the circularity estimates $C_{m}$ computed from the magnetometer data using algorithms 1 and 2 are different in absolute numbers from the real geometric circularity measures computed from the GPS data.

\section{RESULTS AND DISCUSSION}

In personal health services focused on lifestyle behavior change it is important to be able to address the current activities correctly in providing feedback, motivation, and advices. Often the only information source is sensor data, for example, from a wearable device, which has little semantic context. In this paper we study the possibility to get additional information about the activities of a subject from the path trajectories. It is assumed that ambulatory trajectories can be divided into utilitarian and leisure trips based on the whether a subject returns the same path from a trip from A to B, or encloses an area by a loop, respectively. In particular, a measure, circularity, is proposed which characterizes the
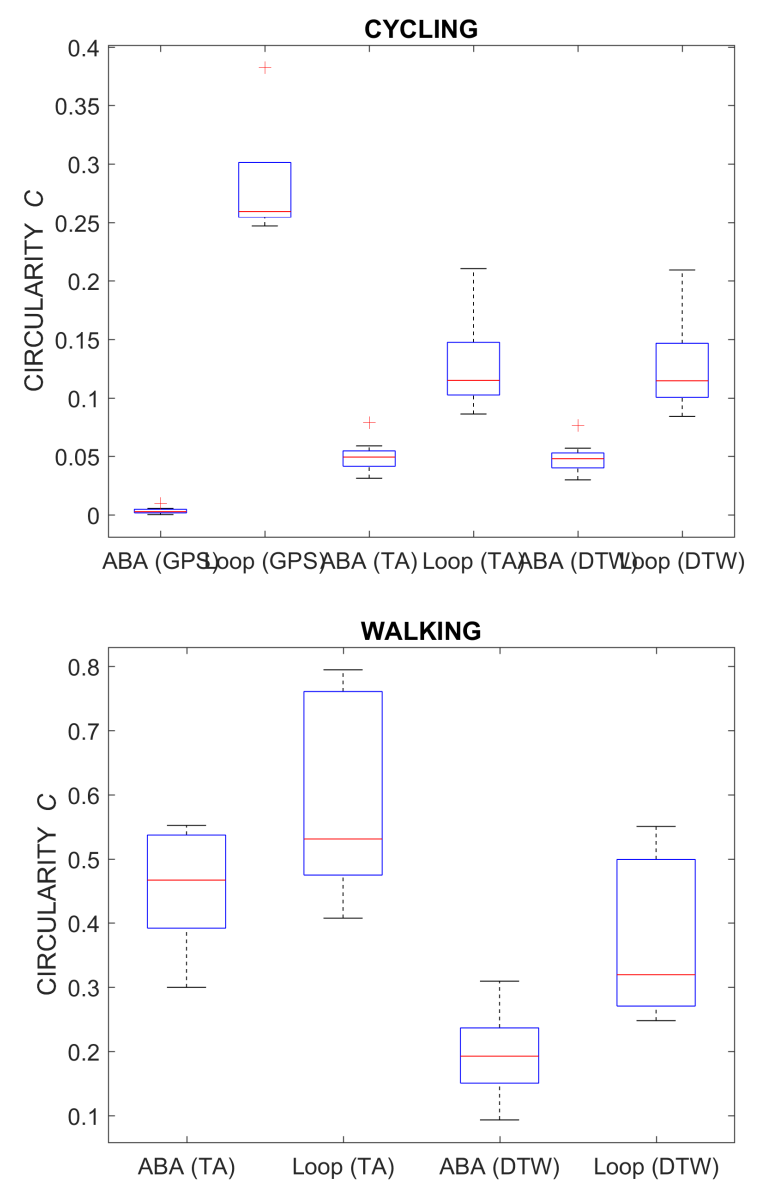

Fig. 4: The estimate of the circularity computed from GPS data, linear time-alignment (TA) and DTW in two-way trips (ABA) and loops in cycling (top) and walking (bottom) data, respectively. GPS data was not available with the walking data.

overall geometric property of the trip as a ratio of the enclosed area and a square of the path length.

It is first demonstrated that the histograms of the proposed measure in walking, running, and cycling trips in sensor data from a population of 85 volunteers look plausible. Secondly, the measure is computed from geographic location tracking data of a collection of cycling and walking paths, which represent either utilitarian or leisure trips.

In many cases real geographic location data is not available and therefore the estimation of the circularity becomes impossible. However, one may note that the lower margin of the circularity can be found even in the case where the subject on a trip from A to B and back returns the same path. Finding a way back is a common phenomenon in many biological organisms which do not have any means of global positioning. For example, the local magnetic signature is related to the environment or direction of movement is known to be used by many animal species including migratory birds and fishes. The proposed algorithm can be seen as an imitation of the process of reverse navigation based on local magnetic cues. 
In the paper we demonstrate that the algorithm in application to the cycling and walking data of utilitarian and leisure trips shows a significant difference in the estimated lower bound for the circularity.

\section{REFERENCES}

[1] Z. Guo and B. P. Y. Loo, "Pedestrian environment and route choice: evidence from New York City and Hong Kong," Journal of Transport Geography, vol. 28, pp. 124-136, Apr. 2013.

[2] A. W. Agrawal, M. Schlossberg, and K. Irvin, "How Far, by Which Route and Why? A Spatial Analysis of Pedestrian Preference," Journal of Urban Design, vol. 13, no. 1, pp. 81-98, Feb. 2008.

[3] S. A. Schroeder, "We Can Do Better Improving the Health of the American People," New England Journal of Medicine, vol. 357, no. 12, pp. 1221-1228, Sep. 2007.

[4] Y. Takama, W. Sasaki, T. Okumura, C. C. Yu, L. H. Chen, and H. Ishikawa, "Walking Route Recommendation System for Taking a Walk as Health Promotion," in 2015 IEEE/WIC/ACM International Conference on Web Intelligence and Intelligent Agent Technology (WIIAT), vol. 1, Dec. 2015, pp. 556-559.

[5] M. A. Case, H. A. Burwick, K. G. Volpp, and M. S. Patel, "Accuracy of Smartphone Applications and Wearable Devices for Tracking Physical Activity Data," JAMA, vol. 313, no. 6, p. 625, Feb. 2015.

[6] K. R. Evenson, M. M. Goto, and R. D. Furberg, "Systematic review of the validity and reliability of consumer-wearable activity trackers," The International Journal of Behavioral Nutrition and Physical Activity, vol. 12, Dec. 2015.

[7] R. C. Beason, "Mechanisms of Magnetic Orientation in Birds," Integrative and Comparative Biology, vol. 45, no. 3, pp. 565-573, Jun. 2005.

[8] R. A. Holland and B. Helm, "A strong magnetic pulse affects the precision of departure direction of naturally migrating adult but not juvenile birds," Journal of The Royal Society Interface, vol. 10, no. 81, pp. 20121 047-20121 047, Feb. 2013.

[9] N. F. Putman, K. J. Lohmann, E. M. Putman, T. P. Quinn, A. P. Klimley, and D. L. G. Noakes, "Evidence for Geomagnetic Imprinting as a Homing Mechanism in Pacific Salmon," Current Biology, vol. 23, no. 4, pp. 312-316, Feb. 2013.

[10] R. A. Singer, B. D. Abroms, and T. R. Zentall, "Formation of a Simple Cognitive Map by Rats," International Journal of Comparative Psychology, vol. 19, no. 4, Dec. 2006.

[11] J. Haverinen and A. Kemppainen, "A global self-localization technique utilizing local anomalies of the ambient magnetic field," in IEEE International Conference on Robotics and Automation, 2009. ICRA '09, May 2009, pp. 3142-3147.

[12] B. Braden, "The Surveyor's Area Formula," The College Mathematics Journal, vol. 17, no. 4, pp. 326-337, 1986.

[13] Z. Kulpa, "Area and perimeter measurement of blobs in discrete binary pictures," Computer Graphics and Image Processing, vol. 6, no. 5, pp. 434-451, Oct. 1977.

[14] G. I. Wilson, M. D. Holton, J. Walker, M. W. Jones, E. Grundy, I. M. Davies, D. Clarke, A. Luckman, N. Russill, V. Wilson, R. Plummer, and R. P. Wilson, "A new perspective on how humans assess their surroundings; derivation of head orientation and its role in framing the environment," PeerJ, vol. 3, p. e908, Jun. 2015.

[15] B. Kim and S. H. Kong, "A Novel Indoor Positioning Technique Using Magnetic Fingerprint Difference," IEEE Transactions on Instrumentation and Measurement, vol. 65, no. 9, pp. 2035-2045, Sep. 2016.

[16] L. M. Devito and H. Eichenbaum, "Memory for the order of events in specific sequences: contributions of the hippocampus and medial prefrontal cortex," The Journal of Neuroscience: The Official Journal of the Society for Neuroscience, vol. 31, no. 9, pp. 3169-3175, Mar. 2011.

[17] T. Madl, S. Franklin, K. Chen, R. Trappl, and D. Montaldi, "Exploring the Structure of Spatial Representations," PloS One, vol. 11, no. 6, p. e0157343, 2016.

[18] K. K. Paliwal, A. Agarwal, and S. S. Sinha, "A modification over Sakoe and Chiba's dynamic time warping algorithm for isolated word recognition," Signal Processing, vol. 4, no. 4, pp. 329-333, Jul. 1982. 